\title{
ANTEGRADE AND RETROGRADE CARDIOPLEGIA: ALTERNATE OR SIMULTANEOUS?
}

Toshizumi Shirai, MD

Vivek Rao, MD

Richard D. Weisel, MD

John S. Ikonomidis, MD, PhD

Nobuhiko Hayashida, MD

Joan Ivanov, RN, MSc

Susan Carson, AHT

Molly K. Mohabeer, BSc

Donald A. G. Mickle, MD, MSc

\begin{abstract}
Neither antegrade nor retrograde cardioplegic protection provides homogeneous distribution, and a combination may be required to avoid anaerobic metabolism and depressed postoperative ventricular function. Tepid cardioplegia $\left(29^{\circ} \mathrm{C}\right)$ avoids the delayed recovery of cardiac function and metabolism associated with cold cardioplegia $\left(15^{\circ} \mathrm{C}\right)$ and reduces the anaerobic metabolism seen with warm $\left(37^{\circ} \mathrm{C}\right)$ cardioplegia. We compared two techniques that combine antegrade and retrograde tepid cardioplegia: alternate and simultaneous. Methods: Sixty patients undergoing elective isolated coronary artery bypass grafting were randomized to receive near continuous tepid retrograde and either intermittent antegrade cardioplegia (the alternate technique) or antegrade cardioplegia with the solution delivered concurrently through each completed vein graft (the simultaneous technique). Results: Myocardial lactate extraction was greater after crossclamp release following simultaneous than alternate cardioplegia. Postoperative ventricular function was better after alternate than simultaneous cardioplegia. Conclusion: Both techniques permitted rapid postoperative recovery of myocardial metabolism and ventricular function. However, simultaneous cardioplegia was simpler and did not require deairing the aortic root between antegrade infusions. (J Thorac Cardiovasc Surg 1996;112:787-96)
\end{abstract}

mprovements in myocardial protection have contributed to the reduction in the morbidity and mortality of cardiac operations. ${ }^{1}$ Continuous warm cardioplegia offered the promise of resuscitating the ischemic myocardium. ${ }^{2}$ Unfortunately coronary obstructions and arterial grafts limit warm blood cardioplegic delivery by the antegrade route. ${ }^{3}$ Retro-

From the Division of Cardiovascular Surgery, Department of Clinical Biochemistry, and the Center for Cardiovascular Research, The Toronto Hospital and the University of Toronto, Toronto, Ontario, Canada.

Supported by the Medical Research Council of Canada (grant MT9825). J.S.I. is a Fellow of the Heart and Stroke Foundation of Canada; V.R. is a Pharmaceutical Roundtable Fellow of the Heart and Stroke Foundation of Canada; R.D.W. is a Career Investigator of the Heart and Stroke Foundation of Ontario.

Presented to the American Heart Association, Anaheim, Calif., November, 1995.

Received for publication Nov. 27, 1995; revisions requested Jan. 16, 1996; revisions received Feb. 13, 1996; accepted for publication Feb. 21, 1996.

Address for reprints: Richard D. Weisel, MD, The Toronto Hospital, 200 Elizabeth St., EN 14-215, Toronto, Ontario, M5G 2C4, Canada.

Copyright $(1996$ by Mosby-Year Book, Inc.

$0022-5223 / 96 \$ 5.00+0 \quad \mathbf{1 2 / 1 / 7 2 9 0 3}$ grade cardioplegia permits delivery beyond an occluded left anterior descending coronary artery when a left internal thoracic artery is used to bypass this vessel. ${ }^{4-6}$ However, venovenous shunts and thebesian channels draining into the ventricular cavity limit distribution of cardioplegic solution with retrograde cardioplegia. ${ }^{4-6}$ Most cardiac surgeons have used a combination of antegrade and retrograde delivery. $^{7}$

Warm cardioplegia permits resuscitation of the ischemic myocardium, which is not possible with cold cardioplegia. ${ }^{4-6}$ Slightly reducing the myocardial temperature reduces myocardial ischemia during cardioplegic interruptions and reducing the systemic temperature may protect the brain and other organs. We found that tepid cardioplegia $\left(29^{\circ} \mathrm{C}\right)$ provided better protection than either warm $\left(37^{\circ} \mathrm{C}\right)$ or cold $\left(15^{\circ} \mathrm{C}\right)$ cardioplegia..$^{8,9}$

Therefore we used tepid cardioplegia to evaluate two techniques of combination cardioplegia to determine which provided better myocardial protection in a prospective randomized trial.

\section{Methods}

Patient population. Sixty men scheduled for isolated coronary artery bypass grafting by one surgeon agreed to 
Table I. Clinical data

\begin{tabular}{lccc}
\hline & & & $p$ \\
& Alternate & Simultaneous & Value \\
\hline Patients & 30 & 30 & \\
Age (yr) & $60.2 \pm 1.6(3)$ & $69.9 \pm 1.4(3)$ & 0.87 \\
NYHA class (II/III/IV) & $3 / 16 / 11$ & $3 / 10 / 17$ & 0.26 \\
Left main disease (Y/N) & $6 / 24$ & $6 / 24$ & 1.00 \\
LVEF (>0.6/0.4-0.6/<0.4) & $11 / 19 / 0$ & $9 / 17 / 4$ & 0.16 \\
Urgent operation & 0 & 0 & 1.00 \\
Grafts (2/3/4/5/6) & $1 / 4 / 20 / 5 / 0$ & $0 / 3 / 18 / 8 / 1$ & 0.57 \\
XCL time (min) & $61.3 \pm 2.4$ & $65.2 \pm 2.8$ & 0.30 \\
CPB time (min) & $80.2 \pm 2.9$ & $84.7 \pm 2.8$ & 0.26 \\
\hline
\end{tabular}

Continuous variables are presented as the mean \pm standard error and were compared by ANOVA. $\chi^{2}$ or Fisher's exact tests were used for categoric variables, $N Y H L A$, New York Heart Association class; $L V E F$, left ventricular ejection fraction; urgent operation, operation within 48 hours of cardiac catheterization or an ischemic event; $X C L$, aortic crossclamp; $C P B$, cardiopulmonary bypass.

participate in this study. All patients signed a consent form approved by our institutional review board. Patients admitted to the study were 40 to 79 years old, had double or triple vessel coronary artery disease, and had adequate preoperative ventricular function (left ventricular ejection fraction $>30 \%$ according to preoperative single-plane contrast ventriculography). Table I describes the patient characteristics.

Operative technique. Cardiopulmonary bypass was established with a single two-stage right atrial cannula. During bypass, the hematocrit value was maintained between $20 \%$ and $25 \%$, pump flows between 2.0 and 2.5 $\mathrm{L} / \mathrm{min}$ per square meter, and mean arterial pressure between 50 and $60 \mathrm{~mm} \mathrm{Hg}$, with administration of sodium nitroprusside or phenylephrine hydrochloride as required. Patients were not actively cooled and their systemic (nasopharyngeal) temperature was allowed to drift to $33^{\circ} \pm 1^{\circ} \mathrm{C}$. Rewarming was accomplished during construction of the last three anastomoses. All distal and proximal anastomoses were performed during a single crossclamp period. The left internal thoracic artery was anastomosed to the left anterior descending coronary artery as the last graft in all patients.

Cardioplegia groups. Patients were randomly assigned, by a computer-generated randomization table, to one of two cardioplegic strategies: alternate or simultaneous. Blood cardioplegic solution was prepared by mixing four parts of oxygenated blood to one part of a crystalloid solution ${ }^{5}$ and was delivered by means of the Buckberg-Shiley Plus system (Sorin Biomedica, Irvine, Calif.). The cardioplegic techniques are depicted in Fig. 1. In both groups, cardiac arrest was achieved with an antegrade infusion of $500 \mathrm{ml}$ highpotassium $(27 \mathrm{mEq} / \mathrm{L})$ cardioplegic solution delivered into the aortic root at a pressure of $70 \mathrm{~mm} \mathrm{Hg}$ measured through a separate port of the aortic root cardioplegia cannula (Research Medical, Inc., Midvale, Utah). ${ }^{4-9}$ The cardioplegic solution was not cooled but the temperature was allowed to drift during the crossclamp period.

Near continuous retrograde and intermittent antegrade cardioplegia (alternate: $n=30$ ). After cardiac arrest was achieved with the initial antegrade infusion, the aortic root was vented and retrograde delivery of low-potassium
$(13 \mathrm{mEq} / \mathrm{L})$ cardioplegic solution was commenced into the coronary sinus cannula (Research Medical) at $200 \mathrm{ml} / \mathrm{min}$. Coronary sinus pressure was monitored continuously by a separate pressure-monitoring port and was maintained below $40 \mathrm{~mm} \mathrm{Hg}$ throughout the procedure. The adequacy of cannula positioning was confirmed by observing distention of the posterior interventricular vein, maintaining the coronary sinus pressure, and palpating the position of the coronary sinus cannula. Distal and proximal anastomoses were constructed sequentially. The retrograde cardioplegic infusion was interrupted whenever necessary to ensure adequate visualization of the coronary artery during each distal anastomosis. Antegrade cardioplegic infusions $(250 \mathrm{ml})$ were given intermittently into the aortic root at a flow rate sufficient to maintain the aortic root pressure at $50 \mathrm{~mm} \mathrm{Hg}$ after completion of each proximal saphenous vein graft anastomosis. The air that accumulated in the aortic root during the construction of the proximal anastomosis was removed before each antegrade aortic root infusion. After the distal anastomosis of the last saphenous vein graft, the left internal thoracic artery was anastomosed to the left anterior descending artery and perfused by noncardioplegic blood. Then the last saphenous vein proximal anastomosis was performed. Cardioplegic infusions were never given simultaneously by the antegrade and retrograde routes.

Near continuous retrograde and simultaneous antegrade vein graft cardioplegia (simultaneous: $n=30$ ). After cardiac arrest, cardioplegic solution was infused retrogradely at $200 \mathrm{ml} / \mathrm{min}$. Cardioplegic infusions were interrupted when necessary to permit adequate coronary visualization. After the completion of each distal anastomosis, each vein graft was attached to a manifold to permit cardioplegic delivery through each completed vein graft and into the coronary sinus from a single pump head. During subsequent distal anastomoses, cardioplegic solution was administered into the coronary sinus and into each completed vein graft with the aortic root vented. Proximal anastomoses were performed after the left internal thoracic artery graft was opened.

The pressure measured in the coronary sinus during retrograde cardioplegia or in the aortic root during antegrade cardioplegia was recorded and used to calculate coronary vascular resistance. The total amount of cardioplegic solution was the same in the two groups, but flow rates were greater in the simultaneous group to compensate for the greater flows during alternate antegrade cardioplegia.

Coronary vascular resistance. Coronary vascular resistance during cardioplegic arrest was calculated as follows

$$
\mathrm{CVR}=\mathrm{CP} \text { pressure } / \mathrm{CP} \text { flow }(\mathrm{mm} \mathrm{Hg} \cdot \mathrm{min} / \mathrm{L})
$$

where CVR is coronary vascular resistance, $\mathrm{CP}$ pressure is aortic root pressure (during antegrade cardioplegia) or coronary sinus pressure (during retrograde cardioplegia).

Oxygen, lactate, and $\mathbf{p H}$ assay. Arterial and coronary venous blood samples were obtained simultaneously during bypass before application of the crossclamp, immediately after crossclamp release, 5 and 10 minutes after crossclamp removal, and 5 and 10 minutes after discontinuing cardiopulmonary bypass. 

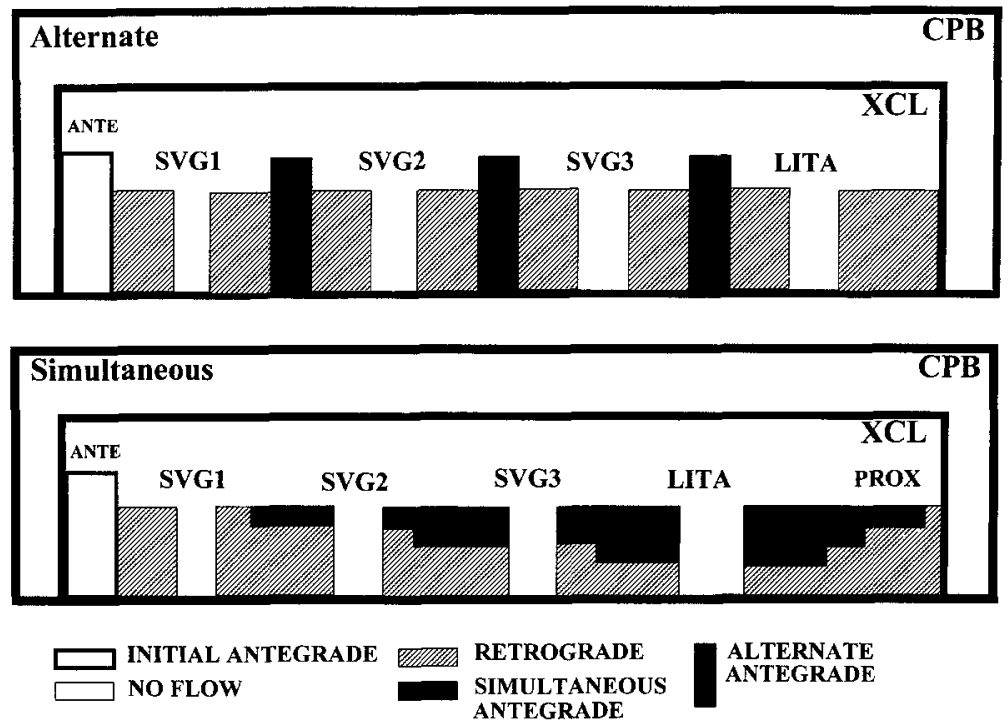

Fig. 1. Cardioplegic technique. The techniques of alternate and simultaneous antegrade cardioplegia during semicontinuous retrograde cardioplegia are depicted. $C P B$, Cardiopulmonary bypass; $X C L$, aortic crossclamp; $S V G$, saphenous vein graft; LITA, left internal thoracic artery graft; PROX, proximal anastomoses of vein grafts; $A N T E$, initial antegrade delivery.

In the alternate group, during crossclamp, cardioplegic and coronary venous blood samples were obtained immediately before the completion of each proximal anastomosis during retrograde cardioplegia and 30 seconds after the initiation of intermittent antegrade cardioplegia. Coronary venous blood samples were taken from the coronary sinus during antegrade delivery of cardioplegic solution and from the aortic root during retrograde delivery.

In the simultaneous group, cardioplegic and coronary venous blood samples were obtained immediately before the completion of each distal anastomosis and 30 seconds after the initiation of antegrade infusions of cardioplegic solution via vein grafts. Coronary venous samples were taken from the aortic root.

Blood samples were assayed for the partial pressure of oxygen $\left(\mathrm{PO}_{2}\right)$ and carbon dioxide $\left(\mathrm{PCO}_{2}\right), \mathrm{pH}$ (Acid-Base Laboratory, Radiometer A/S, Copenhagen, Denmark), and oxygen saturation (Co-Oximeter, Instrumentation Laboratories, Inc., Lexington, Mass.). Oxygen content $\left(\mathrm{O}_{2}\right.$ Con $)$ was calculated from the formula: $\mathrm{O}_{2}$ Con $=$ $1.39 \mathrm{Hgb} \times \mathrm{Sat}+0.0031 \times \mathrm{Po}_{2}$, where $\mathrm{Hgb}$ is the hemoglobin concentration and Sat is the oxygen saturation. Myocardial oxygen extraction was calculated as the arterial or cardioplegic oxygen content minus the coronary venous oxygen content. Measurements were made at $37^{\circ} \mathrm{C}$ and corrected to the myocardial temperature at the time of sampling, which was measured in the left anterior descending region with a temperature probe (Sorin Biomedica).

Blood samples for lactate determination were mixed with a measured volume of $6 \%$ perchloric acid. Lactate concentration was measured in the protein-free supernatant by an enzymatic method (Rapid Lactate Stat Pack kit, Calbiochem-Behring, La Jolla, Calif.). Myocardial lactate extraction was calculated as the difference between arte- rial or cardioplegic and coronary venous lactate content. Negative lactate extraction was expressed as lactate production.

The concentration of hydrogen ion $\left(\left[\mathrm{H}^{+}\right]\right)$in the blood sample was determined by converting the measured $\mathrm{pH}$ value to $\left[\mathrm{H}^{+}\right]$by the formula: $\left[\mathrm{H}^{+}\right]=$antilog $(-\mathrm{pH})$. Measurements were made at $37^{\circ} \mathrm{C}$ and corrected to the myocardial temperature at the time of sampling. Myocardial acid production was calculated as the coronary venous effluent $\left[\mathrm{H}^{+}\right]$minus the arterial or cardioplegic $\left[\mathrm{H}^{+}\right]$.

During cardioplegic arrest, myocardial consumption of oxygen, lactate release, (LR) and acid release were calculated as cardioplegic flow multiplied by the difference between the arterial and coronary venous content.

Hemodynamic measurements. Heart rate (HR), mean arterial blood pressure (MAP), mean pulmonary artery pressure (MPA), mean right atrial pressure (RAP), and pulmonary capillary wedge pressure (PCWP) were measured. Cardiac output (CO) was measured in triplicate by means of a thermodilution technique by an independent observer who was unaware of the cardioplegic technique used. Derived hemodynamic indices were calculated as follows:

$$
\mathrm{CI}=\mathrm{CO} / \mathrm{BSA}\left(\mathrm{L} / \mathrm{min} / \mathrm{m}^{2}\right)
$$

$$
\mathrm{SI}=\mathrm{CI} / \mathrm{HR}(\mathrm{ml} / \mathrm{min} \text { per square meter })
$$

$$
\begin{gathered}
\text { LVSWI }=\text { SI } \times(\mathrm{MAP}-\mathrm{PCWP}) \times 0.0316\left(\mathrm{gm} \cdot \mathrm{m} / \mathrm{m}^{2}\right) \\
\mathrm{RVSWI}=\mathrm{SI} \times(\mathrm{MPA}-\mathrm{RAP}) \times 0.0136\left(\mathrm{gm} \cdot \mathrm{m} / \mathrm{m}^{2}\right) \\
\mathrm{SVRI}=(\mathrm{MAP}-\mathrm{RAP}) \times 80 / \mathrm{CI}\left(\mathrm{dyne} \cdot \mathrm{sec} / \mathrm{cm}^{-5}\right) \\
\mathrm{PVRI}=(\mathrm{MPA}-\mathrm{PAWP}) \times 80 / \mathrm{CI}\left(\text { dyne } \cdot \mathrm{sec} / \mathrm{cm}^{-5}\right)
\end{gathered}
$$


where CI is cardiac index, BSA is body surface area, SI is stroke index, LVSWI is left ventricular stroke work index, RVSWI is right ventricular stroke work index, SVRI is systemic vascular resistance index, PVRI is pulmonary vascular resistance index, and PAWP is pulmonary arterial wedge pressure.

These hemodynamic variables were measured before initiation of cardiopulmonary bypass and at 1, 4, 12, and 24 hours after discontinuation of cardiopulmonary bypass. Postoperative volume repletion followed a standard protocol and was accomplished by individuals who were unaware of the intraoperative cardioplegic technique used.

Creatine kinase measurement. An antibody inhibition technique was employed to measure the $\mathrm{MB}$ isozyme of creatine kinase (CK-MB). Sequential CK-MB measurements were performed at 2, 4, 8, 16, 24, and 48 hours after crossclamp release. Integration of the area under the concentration-time curve for CK-MB within the first 48 hours after the operation allowed calculation of the total CK-MB release, expressed as units times hours divided by liters. $^{5}$

Statistical analysis. Statistical analysis was performed with the SAS program (SAS Institute, Inc., Cary, N.C.). One-way or two-way analyses of variance (ANOVA) were used to test the effect of cardioplegic group and time on myocardial oxygen use, lactate and acid release, coronary vascular resistance, hemodynamic variables, and postoperative CK-MB release. When the ANOVA indicated a significant effect of cardioplegic group or time $(p<0.05)$, the differences were specified with Duncan's multiple range test. The relation between cardiac index left ventricular stroke work index, right ventricular stroke work index, and the corresponding preload were evaluated by an analysis of covariance. Categoric data are displayed as the absolute and percent frequency. Continuous variables are listed as the mean and standard error of the mean. Statistical significance was assumed at a probability level of less than 0.05 .

\section{Results}

Myocardial temperature. Mean myocardial temperatures during cardioplegic arrest in the territory of the left anterior descending coronary artery were similar between groups (alternate, $29.2^{\circ} \pm 0.4^{\circ} \mathrm{C}$; simultaneous, $28.7^{\circ} \pm 0.5^{\circ} \mathrm{C} ; p=0.62$ ).

Cardioplegic flow rates and continuity. The initial antegrade cardioplegic flow rates were similar between groups (alternate, $302 \pm 13 \mathrm{ml} / \mathrm{min}$; simultaneous, $310 \pm 12 \mathrm{ml} / \mathrm{min} ; p=0.66)$. Total cardioplegic volume and the cardioplegic flow rates were similar between groups (total volume: alternate, $6082 \pm 305 \mathrm{ml}$; simultaneous, $6335 \pm 366 \mathrm{ml}$; $p=0.60$; flow rate: alternate, $129 \pm 7 \mathrm{ml} / \mathrm{min}$; simultaneous, $130 \pm 7 \mathrm{ml} / \mathrm{min} ; p=0.96)$. The percentage of crossclamp time during which cardioplegia was interrupted was similar between the two groups (alternate, $13 \% \pm 1 \%$; simultaneous, $14 \% \pm$ $1 \% ; p=0.41)$.
Coronary vascular resistance. The aortic root pressure and the coronary vascular resistance were similar between the groups during the first antegrade infusion (aortic root pressure: alternate, $67 \pm$ $4 \mathrm{~mm} \mathrm{Hg}$; simultaneous, $66 \pm 3 \mathrm{~mm} \mathrm{Hg} ; p=0.76$; coronary vascular resistance: alternate, $204 \pm 26$ $\mathrm{mm} \mathrm{Hg} \cdot \min / \mathrm{L} ;$ simultaneous, $189 \pm 16 \mathrm{~mm}$ $\mathrm{Hg} \cdot \min / \mathrm{L} / p=0.61$ ).

During retrograde delivery of cardioplegic solution, coronary vascular resistance was $142 \pm 15 \mathrm{~mm}$ $\mathrm{Hg} \cdot \mathrm{min} / \mathrm{L}$ in the alternate group and $139 \pm 17 \mathrm{~mm}$ $\mathrm{Hg} \cdot \mathrm{min} / \mathrm{L}$ in the simultaneous group $(p=0.89)$.

Myocardial metabolism during cardioplegia. Myocardial oxygen consumption and lactate and acid release at 10,30 , and 50 minutes of the crossclamp period are shown in Fig. 2.

Myocardial oxygen consumption was significantly greater (group $p=0.0001$ by ANOVA) during intermittent antegrade cardioplegia than during continuous retrograde cardioplegia in the alternate group. The difference probably resulted from contamination of the aortic root samples obtained during retrograde cardioplegia by bronchial arterial blood inducing an underestimation of oxygen consumption and lactate and acid release during retrograde cardioplegia. ${ }^{10}$

Oxygen consumption during simultaneous cardioplegia tended to be higher than during alternate cardioplegia, but the difference did not reach statistical significance (group $p=0.079$ by ANOVA).

Myocardial lactate released during cardioplegia was higher with simultaneous retrograde and vein graft cardioplegia than with continuous retrograde cardioplegia in the alternate group (group $p=0.005$ by ANOVA).

Myocardial acid release with simultaneous cardioplegia was similar to that with alternate cardioplegia (group $p=0.85$ by ANOVA). Acid release with intermittent antegrade cardioplegia was greater than with continuous retrograde cardioplegia (group $p=0.007$ by ANOVA) in the alternate group. Acid release increased with time in both the alternate and simultaneous groups (time: alternate, $p=0.03$; simultaneous, $p=0.03$ by ANOVA).

Reperfusion. Thirteen patients in the alternate group and 11 patients in the simultaneous group required atrial or atrioventricular sequential pacing after discontinuation of cardiopulmonary bypass $(p=0.59)$. However, only two patients in the simultaneous group required pacing at the time of return to the intensive care unit, whereas 10 patients in the alternate group still required pacing ( $p=$ 

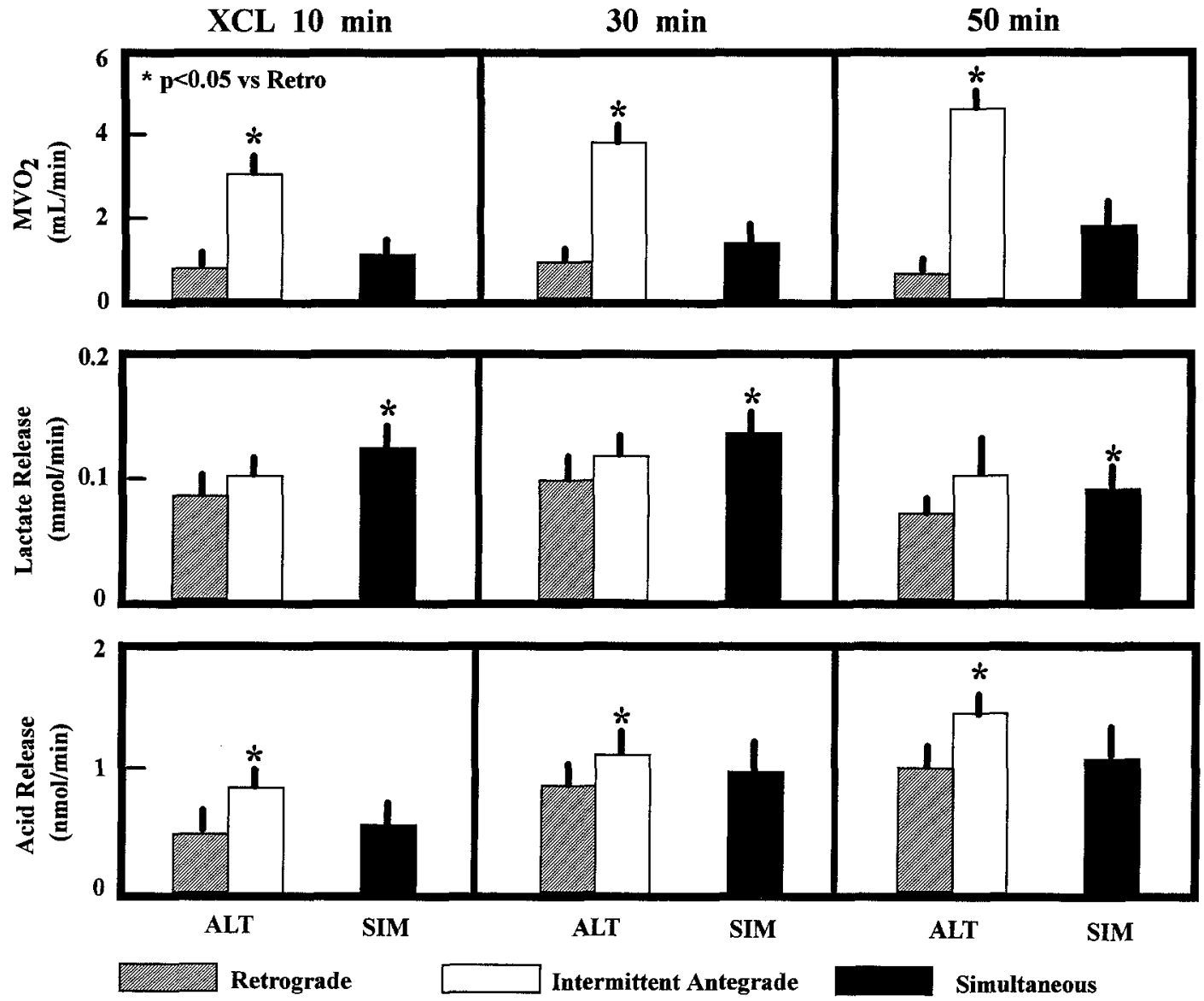

Fig. 2. Myocardial metabolism during cardiac arrest. Myocardial oxygen consumption $\left(\mathrm{MVO}_{2}\right)$, lactate release, and acid release $(A R)$ at 10,30 , and 50 minutes of crossclamp time are illustrated. Oxygen consumption was greater during intermittent antegrade cardioplegia in the alternate group $(p<0.001$ by ANOVA). Myocardial lactate release was greater with simultaneous cardioplegia than with continuous retrograde cardioplegia in the alternate group ( $p=0.005$ by ANOVA). Myocardial acid release was greatest with intermittent antegrade cardioplegia $(p=0.007$ by ANOVA).

0.009). No patient required pacing 4 hours after the operation.

The highest serum potasium level during cardiopulmonary bypass was similar between the two groups (alternate, $6.11 \pm 0.64 \mathrm{mEq} / \mathrm{L}$; simultaneous, $6.37 \pm 0.52 \mathrm{mEq} / \mathrm{L} ; p=0.084)$ and at discontinuation of cardiopulmonary bypass (alternate, $5.93 \pm$ $0.63 \mathrm{mEq} / \mathrm{L}$; simultaneous, $5.95 \pm 0.49 \mathrm{mEq} / \mathrm{L} ; p=$ $0.910)$. No differences were detected between the groups in mean arterial pressure $(p=0.51$ by ANOVA) or nasopharyngeal temperature ( $p=0.72$ by ANOVA) at any time before, during, or after cardioplegia.

Myocardial metabolism during reperfusion. Myocardial oxygen extraction and lactate and acid production before crossclamping, immediately, 5, and 10 minutes after aortic crossclamp removal, and 5 and 10 minutes after discontinuation of cardiopulmonary bypass are depicted in Fig. 3 .

Myocardial oxygen extraction was similar between groups ( $p=0.15$ by ANOVA) during reperfusion.

Immediately after removal of the crossclamp, lactate production was similar in the two groups $(p=0.93)$. In the simultaneous group, lactate extraction recovered more quickly than in the alternate group (interaction between group and time $p=$ 0.03 by ANOVA). Lactate extraction was greater in the simultaneous group at 10 minutes during cardiopulmonary bypass and 10 minutes after cardiopulmonary bypass.

Acid production during reperfusion was similar between groups ( $p=0.55$ by ANOVA). 

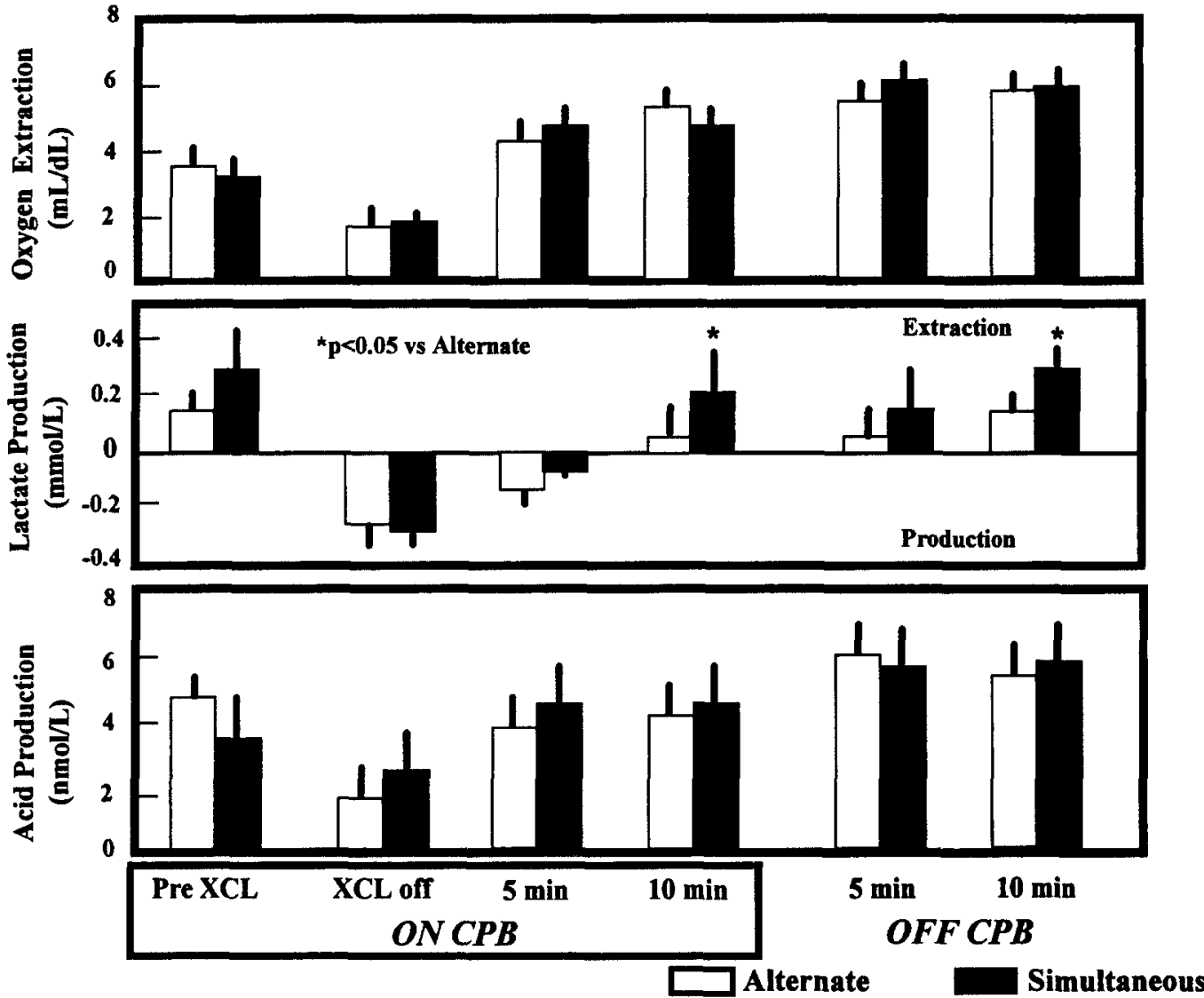

Fig. 3. Myocardial metabolism during reperfusion. Myocardial oxygen extraction, lactate extraction, and acid release before aortic crossclamping, immediately, 5 , and 10 minutes after crossclamp removal, and 5 and 10 minutes after discontinuation of cardiopulmonary bypass are illustrated. Lactate extraction recovered more quickly in the simultaneous group than in the alternate group ( $p=0.03$ by ANOVA), and lactate extraction was greater 10 minutes on cardiopulmonary bypass and 10 minutes after cardiopulmonary bypass. No differences in oxygen extraction or acid release were detected between groups.

Hemodynamic measurements. The hemodynamic measurements before and after cardiopulmonary bypass are summarized in Table II. No differences between groups were detected before cardiopulmonary bypass.

Heart rate increased in the postoperative period in both groups (time $p=0.0001$ ). Mean arterial pressure decreased after the operation in both groups compared with prebypass pressure (time $p=$ 0.025 in the alternate group, $p=0.0041$ in the simultaneous group), but there were no differences between groups $(p=0.41)$.

Cardiac index increased after the operation in both groups (time $p=0.0001$ by ANOVA), but there was no difference between groups ( $p=0.20$ by ANOVA).
Pulmonary capillary wedge pressure decreased after cardiopulmonary bypass (time $p=0.017$ by ANOVA) but was not different between the groups ( $p=0.62$ by ANOVA).

Systemic vascular resistance index decreased after the operation (interaction between time and group $p=0.0001$ in both groups) and was not different between groups ( $p=0.65$ by ANOVA).

Left ventricular stroke work index was greater in the alternate group than the simultaneous group (interaction between time and group $p=0.04$ by ANOVA). In addition, stroke work index decreased after the operation in the simultaneous group ( $p=$ $0.02)$ but not in the alternate group $(p=0.26)$.

Mean pulmonary artery pressure was similar between groups ( $p=0.51$ by ANOVA). Right atrial 
Table II. Hemodynamics

\begin{tabular}{|c|c|c|c|c|c|c|}
\hline & \multirow[b]{2}{*}{ Group } & \multicolumn{5}{|c|}{ Time of study } \\
\hline & & Pre- $C P B$ & $1 \mathrm{hr}$ & $4 h r$ & $12 \mathrm{hr}$ & $24 h r$ \\
\hline \multicolumn{7}{|l|}{ Systemic } \\
\hline HR & Alt & $63 \pm 2$ & $80 \pm 2^{*}$ & $80 \pm 2^{*}$ & $85 \pm 2^{*}$ & $85 \pm 2^{*}$ \\
\hline (beats/min) & Simul & $64 \pm 2$ & $80 \pm 2^{*}$ & $87 \pm 2^{*}+$ & $89 \pm 2^{*}$ & $88 \pm 2^{*}$ \\
\hline MAP & Alt & $80.6 \pm 2.1$ & $72.8 \pm 1.8^{*}$ & $77.7 \pm 1.8$ & $74.3 \pm 1.7^{*}$ & $74.7 \pm 2.0^{*}$ \\
\hline$(\mathrm{mm} \mathrm{Hg})$ & Simul & $79.6 \pm 2.2$ & $70.2 \pm 1.5^{*}$ & $75.6 \pm 1.7$ & $73.8 \pm 1.7^{*}$ & $76.7 \pm 1.8$ \\
\hline CI & Alt & $2.3 \pm 0.1$ & $2.8 \pm 0.1^{*}$ & $2.6 \pm 0.1^{*}$ & $3.0 \pm 0.1^{*}$ & $2.8 \pm 0.1^{*}$ \\
\hline$\left(\mathrm{L} / \mathrm{min} / \mathrm{m}^{2}\right)$ & Simul & $2.2 \pm 0.1$ & $2.7 \pm 0.1^{*}$ & $2.5 \pm 0.1$ & $2.8 \pm 0.1^{*}$ & $2.8 \pm 0.1^{*}$ \\
\hline PCWP & Alt & $10.7 \pm 0.8$ & $9.6 \pm 0.6$ & $10.9 \pm 0.6$ & $9.0 \pm 0.8$ & $10.3 \pm 0.7$ \\
\hline$(\mathrm{mm} \mathrm{Hg})$ & Simul & $9.9 \pm 0.5$ & $8.1 \pm 0.6^{*}$ & $11.9 \pm 1.0$ & $10.7 \pm 0.7$ & $11.2 \pm 0.8$ \\
\hline LVSWI & Alt & $35.0 \pm 1.9$ & $31.2 \pm 1.9$ & $29.8 \pm 1.4$ & $32.1 \pm 2.1$ & $30.0 \pm 2.0$ \\
\hline$\left(\mathrm{gm} \cdot \mathrm{m} / \mathrm{m}^{2}\right)$ & Simul & $34.3 \pm 2.3$ & $28.8 \pm 1.4^{*}$ & $25.7 \pm 1.2^{*}$ & $27.5 \pm 1.4^{*}$ & $28.4 \pm 1.1^{*}$ \\
\hline SVRI & Alt & $2699 \pm 158$ & $1929 \pm 95^{*}$ & $2194 \pm 83^{*}$ & $1885 \pm 74^{*}$ & $1910 \pm 62^{*}$ \\
\hline$\left(\right.$ dyne $\left.\cdot \mathrm{sec} \cdot \mathrm{cm}^{-5}\right)$ & Simul & $2680 \pm 122$ & $1972 \pm 93^{*}$ & $2172 \pm 88^{*}$ & $1950 \pm 77^{*}$ & $2028 \pm 86^{*}$ \\
\hline \multicolumn{7}{|l|}{ Pulmonary } \\
\hline MPAP & Alt & $15.6 \pm 0.9$ & $14.4 \pm 0.7$ & $15.3 \pm 0.6$ & $13.8 \pm 0.8$ & $15.4 \pm 0.8$ \\
\hline$(\mathrm{mm} \mathrm{Hg})$ & Simul & $15.6 \pm 0.7$ & $12.9 \pm 0.6^{*}$ & $16.1 \pm 1.1$ & $15.7 \pm 0.8$ & $16.3 \pm 0.9$ \\
\hline $\mathrm{RAP}$ & Alt & $7.55 \pm 0.69$ & $7.90 \pm 0.45$ & $8.40 \pm 0.49$ & $6.77 \pm 0.72$ & $8.00 \pm 0.64$ \\
\hline$(\mathrm{mm} \mathrm{Hg})$ & Simul & $7.86 \pm 0.63$ & $6.37 \pm 0.52$ & $8.50 \pm 0.92$ & $7.25 \pm 0.60$ & $8.12 \pm 0.79$ \\
\hline RVSWI & Alt & $4.09 \pm 0.38$ & $3.04 \pm 0.28$ & $3.12 \pm 0.28$ & $3.39 \pm 0.27$ & $3.34 \pm 0.25$ \\
\hline$\left(\mathrm{gm} \cdot \mathrm{m} / \mathrm{m}^{2}\right)$ & Simul & $3.82 \pm 0.40$ & $3.12 \pm 0.31$ & $3.14 \pm 0.36$ & $3.70 \pm 0.31$ & $3.52 \pm 0.24$ \\
\hline PVRI & Alt & $176 \pm 16$ & $144 \pm 11$ & $140 \pm 10$ & $131 \pm 8$ & $155 \pm 11$ \\
\hline$\left(\right.$ dyne $\left.\cdot \mathrm{sec} \cdot \mathrm{cm}^{-5}\right)$ & Simul & $208 \pm 19$ & $148 \pm 9 *$ & $131 \pm 7^{*}$ & $147 \pm 8^{*}$ & $153 \pm 10^{*}$ \\
\hline
\end{tabular}

The hemodynamic results were evaluated by a two-way ANOVA, and when significant $(p<0.05)$ effects were found, they were specified by Duncan's multiple range test. $H R$, Heart rate; $M A P$, mean arterial pressure; $C I$, cardiac index; $P C W P$, pulmonary capillary wedge pressure; $L V S W I$, left ventricular stroke work index; $S V R I$, systemic vascular resistance index; $M P A P$, mean pulmonary artery pressure; $R A P$, right atrial pressure; $R V S W I$, right ventricular stroke work index; PVRI, pulmonary vascular resistance index; Alt, alternate; Simul, simultaneous.

$* p<0.05$ versus pre-CPB.

$\dagger p<0.05$ versus Alt.

pressures did not change and were not different between groups. Pulmonary vascular resistance index decreased after the operation in the simultaneous group (time $p=0.0001$ ) but not in the alternate group (time $p=0.09$ ), although the groups were not different (interaction between time and group $p=0.41$ ). Right ventricular stroke work indices were not different between groups $(p=0.84$ by ANOVA).

Ventricular function. The relation between left ventricular stroke work index or cardiac index and the corresponding pulmonary wedge pressures are depicted in Fig. 4. The analysis of covariance demonstrated depressed function in the simultaneous group compared with the alternate group (interaction between time and group $p=0.03$ ). Stroke work index was lower in the simultaneous group 4 hours after the operation at higher pulmonary wedge pressures. Cardiac index increased similarly in both groups.

Postoperative release of CK-MB. Postoperative release of CK-MB was not different between the two groups in the first 48 hours (alternate, $1330 \pm 88$
$\mathrm{U} \times \mathrm{hr} / \mathrm{L}$; simultaneous, $1296 \pm 180 \mathrm{U} \times \mathrm{hr} / \mathrm{L} ; p=$ $0.86)$.

Clinical outcome. The clinical outcomes are presented in Table III. None of the 60 patients in this series died. One patient in the simultaneous group had a perioperative myocardial infarction (defined as the appearance of new $\mathrm{Q}$ waves or ischemic ST segment changes with a concomitant rise in CKMB) and low output syndrome developed after the operation (defined as a requirement for inotropic medication because of a cardiac index $<2.1 \mathrm{~L} / \mathrm{min}$ per square meter and systolic blood pressure $<90$ $\mathrm{mm} \mathrm{Hg}$, despite adequate preload and correction of any electrolyte disorders). This patient recovered well and was discharged home on the sixth postoperative day. No patient had a postoperative stroke. No differences between groups were observed.

\section{Discussion}

Improvements in myocardial protection have contributed to the low risks of cardiac operations. ${ }^{3,11,12}$ However, the increasing proportion of patients at high risk being referred for cardiac surgery ${ }^{11,12}$ 

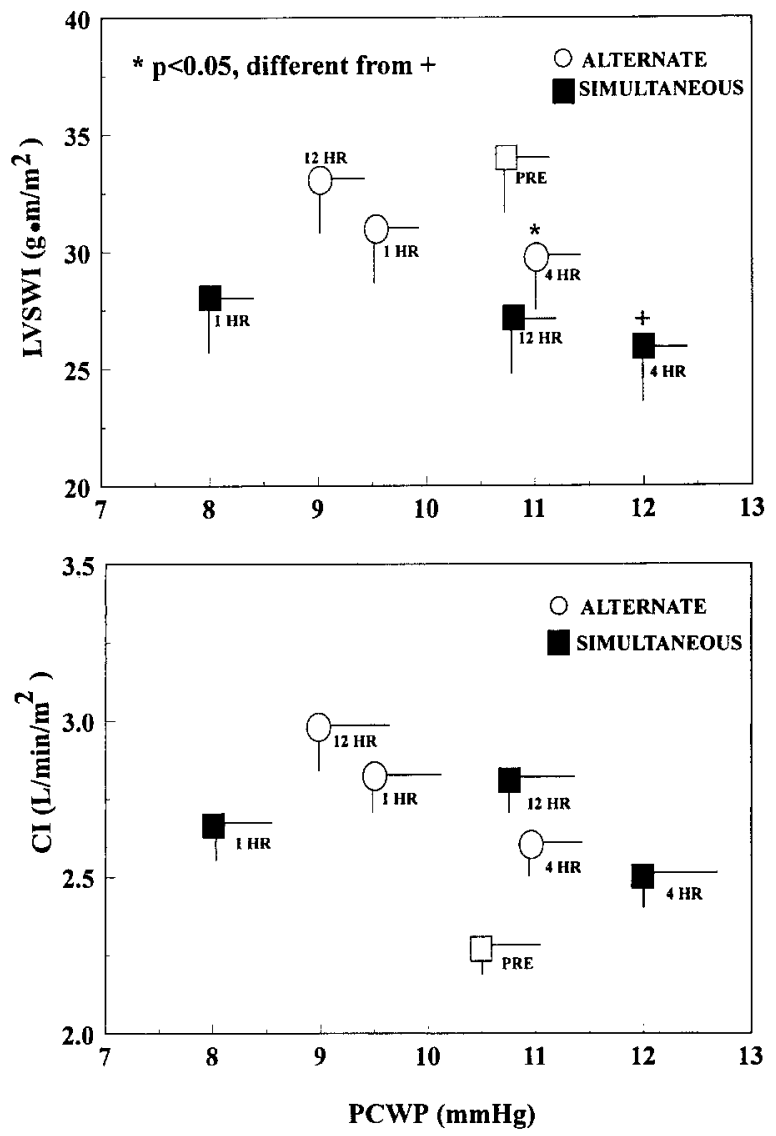

Fig. 4. Postoperative cardiac function. The relation between left ventricular stroke work index (LVSWI) or cardiac index $(C I)$, and pulmonary capillary wedge pressure $(P C W P)$ is depicted. Left ventricular stroke work index decreased in both groups after the operation (time $p<0.0001$ ). The decrease was greater in the simultaneous group than the alternate group ( $p=0.04$ by analysis of covariance). Stroke work index was lower in the simultaneous group 4 hours after the operation at a higher wedge pressure. Cardiac index increased (time $p=0.017$ ) similarly in both groups after the operation.

requires improved techniques of myocardial protection to prevent an increase in morbidity and mortality. The continuous delivery of resuscitative substances offers the best hope of restoring metabolic and contractile function to the ischemic heart. However, the optimal temperature and direction for continuous cardioplegia must be established.

Temperature. Kaukoranta and colleagues ${ }^{13}$ found that mild hypothermic $\left(29^{\circ} \mathrm{C}\right)$ cardioplegia reduced myocardial oxygen consumption, acid, and CK-MB release compared with normothermic $\left(37^{\circ} \mathrm{C}\right)$ cardioplegia. We previously reported that
Table III. Clinical outcomes

\begin{tabular}{lccc}
\hline & Altemate & Simultaneous & $p$ Value \\
\hline Death & 0 & 0 & 1.0 \\
Myocardial infarction & 0 & 1 & 0.3 \\
Low output syndrome & 0 & 1 & 0.3 \\
Intraarotic balloon pump & 0 & 0 & 1.0 \\
Stroke & 0 & 0 & 1.0
\end{tabular}

The $p$ values refer to the results of $\chi^{2}$ test.

tepid cardioplegia reduced oxygen consumption and lactate and acid release to a greater degree than warm cardioplegia. ${ }^{8,9}$ In addition, we found that tepid cardioplegia was associated with better ventricular function 4 hours after the operation than either warm or cold cardioplegia. ${ }^{9}$ In this study CK-MB levels were lower than we have previously reported with either warm or cold cardioplegia. 8,9

Direction. Coronary artery obstructions limit antegrade cardioplegic delivery to ischemic regions of the heart. Retrograde infusions may deliver cardioplegic solution beyond obstructed coronary arteries and may improve subendocardial perfusion. $^{14,15}$ Unfortunately, venovenous shunts and thebesian channels limit cardioplegic distribution to some regions. ${ }^{5-7,16,17}$

Combination cardioplegia. In previous studies we found that retrograde cardioplegia incompletely perfused the heart because of progressive myocardial lactate and acid release during the crossclamp period. ${ }^{5-10}$ We also found that an antegrade infusion washed out lactate that accumulated in the heart during continuous warm retrograde cardioplegia. ${ }^{7-9}$ In this study we found that vein graft infusions increased cardiac lactate release and antegrade infusions increased acid release compared with retrograde infusions. Therefore combination cardioplegia provided more homogeneous perfusion than retrograde cardioplegia. Our findings are similar to those previously published. ${ }^{15,17,1.8}$

Previous studies have established the feasibility of combination cardioplegia. ${ }^{19}$ With both the alternate and simultaneous techniques the aorta root was vented. We avoided delivering cardioplegic solution simultaneously into the coronary sinus and into the aortic root to avoid excessive pressurization and secure rapid egress of any excessive cardioplegic solution.

Both techniques permitted the rapid recovery of aerobic myocardial lactate extraction, but lactate extraction recovered sooner after simultaneous cardioplegia. Ventricular function recovered quickly 
with both techniques but recovery was more rapid after alternate cardioplegia.

This prospective randomized trial demonstrated similar results with both techniques. However, the alternate technique was cumbersome and required careful attention to deairing of the aortic root before intermittent antegrade infusions could be started. Air entered the ascending aorta during construction of each proximal anastomosis and its removal was necessary before each antegrade infusion. The simultaneous technique did not require deairing of the aortic root and permitted completion of the coronary bypass operation without concern for coronary air embolism. Therefore we believe that the simultaneous technique is simpler and has technical advantages.

The two techniques of combination cardioplegia were only marginally different and both required additional maneuvers by the surgeon. However, the additional infusions provide better protection than that achieved by either antegrade or retrograde cardioplegia alone.

Limitations of the study. We found no differences in clinical outcomes between the simultaneous and alternate cardioplegic techniques. This study was not designed to evaluate clinical outcomes, and insufficient power was available to detect clinical differences. The study was designed to detect subtle differences in metabolism and function. We found that the simultaneous technique improved the postoperative recovery of metabolism and the alternate technique improved the postoperative recovery of ventricular function. Therefore sufficient power was available to detect subtle differences, and both techniques effectively protected the heart.

The assessment of myocardial metabolism during retrograde cardioplegia is inaccurate because bronchial collateral blood contaminates the aortic root samples with oxygenated arterial blood, which has a low lactate and acid content. ${ }^{10}$ Therefore myocardial oxygen consumption and lactate and acid release are underestimated during retrograde cardioplegia. ${ }^{10}$ The addition of vein graft infusions during retrograde cardioplegia should further contaminate the aortic root samples and increase the underestimation. The use of a single pump head for retrograde and vein graft infusions may have limited antegrade cardioplegic delivery. However, the greater lactate release during simultaneous cardioplegic arrest suggests even greater lactate washout with vein graft infusions. These reservations do not extend to the assessment of myocardial oxygen extraction and lactate or acid production during reperfusion. The delayed recovery of aerobic lactate extraction after alternate cardioplegia was not limited by contaminated samples.

Our assessment of ventricular function may have been confounded by changes in diastolic compliance. However, we found greater stroke work indices at lower pulmonary capillary wedge pressures after simultaneous cardioplegia. In a previous study we found better ventricular function after warm than cold cardioplegia using load-independent indices of ventricular function. ${ }^{20}$ End-diastolic pressurevolume relations were not altered. Therefore the differences in ventricular function may be independent of the loading conditions.

\section{Summary}

Continuous retrograde cardioplegia was associated with considerable anaerobic lactate accumulation in the myocardium. Both intermittent antegrade infusions and vein graft infusions washed out the lactate and facilitated the recovery of aerobic metabolism and ventricular function. However, the simultaneous technique was more convenient than the alternate technique.

\section{REFERENCES}

1. Kouchoukos NT, Oberman A, Kirklin JW, et al. Coronary bypass surgery: analysis of factors affecting hospital mortality. Circulation 1980;62(Suppl):I84-9.

2. Lichtenstein SV, Ashe KA, El Dalati H, Cusimano RJ, Panos A, Slutsky AS. Warm heart surgery. J Thorac Cardiovasc Surg 1991;101:269-74.

3. Quintillo C, Voci P, Bilotta F, Luzi G, Chiarotte F, Acconcia $\mathrm{C}$, et al. Risk factors of incomplete distribution of cardioplegic solution during coronary artery grafting. J Thorac Cardiovasc Surg 1995;109:439-47.

4. Yau TM, Weisel RD, Mickle DAG, et al. Optimal delivery of blood cardioplegia. Circulation 1991;84(Suppl):III380-8.

5. Yau TM, Weisel RD, Mickle DAG, et al. Alternative techniques of cardioplegia. Circulation 1992;86(Suppl):II377-84.

6. Yau TM, Ikonomidis JS, Weisel RD, et al. Which techniques of cardioplegia prevent ischemia? Ann Thorac Surg 1993;56: 1020-8.

7. Hayashida N, Ikonomidis JS, Weisel RD, Shirai T, Ivanov J, Carson $\mathrm{S}$, et al. Adequate distribution of warm cardioplegia. J Thorac Cardiovasc Surg 1995;110:800-12.

8. Hayashida N, Ikonomidia JS, Weisel RD, et al. The optimal cardioplegic temperature. Ann Thorac Surg 1994;58:961-71.

9. Hayashida N, Shirai T, Weisel RD, et al. Tepid antegrade and retrograde cardioplegia. Ann Thorac Surg 1995;59:723-9.

10. Ikonomidis JS, Yau TM, Weisel RD, Hayashida N, Fu X, Komeda M, et al. Optimal flow rates for warm retrograde cardioplegia. J Thorac Cardiovasc Surg 1994;107:510-9.

11. Christakis GT, Ivanov $\mathbf{J}$, Weisel RD, et al. The changing pattern of coronary artery bypass surgery. Circulation 1989; 80(Suppl):I151-61. 
12. Christakis GT, Weisel RD, Fremes SE, Ivanov J, David TE, Goldman BS, et al. Coronary artery bypass grafting in patients with poor ventricular function. $\mathbf{J}$ Thorac Cardiovase Surg 1992;103:1083-92.

13. Kaukoranta $P$, Lepojarri $M$, Nissinen $J$, Raatikainen $P$, Peuhkurinen KJ. Normothermic versus mild hypothermic retrograde blood cardioplegia: a prospective randomized study. Ann Thorac Surg 1995;60:1087-93.

14. Crooke GA, Harris LJ, Grossi EA, Baumann FG, Galloway $\mathrm{AC}$, Colvin SB. Biventricular distribution of cold blood cardioplegic solution administered by different retrograde techniques. J Thorac Cardiovasc Surg 1991;102:631-7.

15. Partington MT, Acar C, Buckberg CD, Julia P, Kofsky ER, Bgyi HI. Studies of retrograde cardioplegia. I. Capillary blood flow distribution to myocardium supplied by open and occluded arteries. J Thorac Cardiovase Surg 1989;97:605-12.

16. Aronson S, Lee BK, Liddicoat JR, et al. Assessment of retrograde cardioplegia distribution using contrast echocardiography. Ann Thorac Surg 1991;52:810-4.

17. Caldarone Ca, Krukenkamp IB, Misare BD, Levitsky S. Perfusion deficits with retrograde warm blood cardioplegia. Ann Thorac Surg 1994;57:403-6.

18. Stirling MC, McClanahan TB, Schott RJ, Lynch MJ, Bolling $\mathrm{SV}$, Kirsh MM, et al. Distribution of cardioplegic solution infused antegradely and retrogradely in normal canine hearts. J Thorac Cardiovasc Surg 1989;98:1066-76.

19. Ihnken K, Morita K, Buckberg GD, et al. The safety of simultaneous arterial and coronary sinus perfusion: experimental background and initial clinical results. J Card Surg 1194;9:15-25.

20. Yau TM, Ikonomidis JS, Weisel RD, Mickle DAG, Ivanov J, Mohabeer MK, et al. Ventricular function after normothermic versus hypothermic cardioplegia. J Thorac Cardiovasc Surg 1993;105:833-44. 